\title{
When a Diuretic Causes Pulmonary Oedema
}

\author{
Matteo Traversa ${ }^{1}$, Andrea Collini ${ }^{2}$, Paola Villois ${ }^{3}$, Fabrizio Elia ${ }^{4}$, Andrea Verhovez ${ }^{4}$, Franco Aprà ${ }^{4}$ \\ ${ }^{1}$ Internal Medicine, Città della Salute e della Scienza di Torino, Torino, Italy \\ ${ }^{2}$ Emergency Medicine, Città della Salute e della Scienza di Torino, Torino, Italy \\ ${ }^{3}$ Emergency Medicine, San Lazzaro Hospital, Alba, Italy \\ ${ }^{4}$ High Dependency Unit, San Giovanni Bosco Hospital, Torino, Italy
}

\section{Doi: 10.12890/2018_000864-European Journal of Case Reports in Internal Medicine - C EFIM 2018}

\begin{abstract}
Received: $12 / 02 / 2018$
Accepted: 05/03/2018

Published: 05/04/2018
\end{abstract}

How to cite this article: Traversa M, Collini A, Villois P, Elia F, Verhovez A, Aprà F. When a diuretic causes pulmonary oedema. EJCRIM 2018;5: doi:10.12890/2018_000864.

Conflicts of Interests: The Authors declare that there are no competing interests.

This article is licensed under a Commons Attribution Non-Commercial 4.0 License

\section{ABSTRACT}

Background: Hydrochlorothiazide (HCTZ) is one of the most popular drugs for the treatment of hypertension and heart failure. Most of its side effects are harmless and predictable, but some studies report a few life-threatening reactions to this drug, one of the most dangerous being acute pulmonary oedema.

Case Report: A 73-year-old woman was admitted to the Emergency Department with acute respiratory failure due to pulmonary oedema. Her past medical history included long-lasting hypertension with permanent atrial fibrillation and mitral stenosis. Her blood pressure control had been suboptimal, so her cardiologist had changed amlodipine to combination therapy with ramipril and HCTZ. However, 20 min after taking the new drug, the patient experienced fever, vomiting and diarrhoea immediately followed by acute onset of dyspnoea.

Conclusion: Since HCTZ is one of the most popular drugs for hypertension treatment and millions of patients take it every day, it is important to keep in mind both the common adverse reactions as well as the dangerous, although rare, ones.

\section{LEARNING POINTS}

- Pulmonary oedema is a very unusual adverse reaction to hydrochlorothiazide, and a rare presentation of a common condition.

- Pulmonary oedema is not always due to heart problems.

- It is important to keep in mind that hypersensitivity reactions may have many different presentations.

\section{KEYWORDS}

Hydrochlorothiazide, pulmonary edema, inflammatory systemic syndrome, hypersensitivity reaction

\section{INTRODUCTION}

Hydrochlorothiazide (HCTZ) is one of the most popular drugs for the treatment of hypertension. Hypotension, electrolyte abnormalities, orthostatic syncope, hyperglycaemia, photosensitivity and skin exanthema are some of the well-known adverse reactions to thiazide treatment. While most of these side effects are predictable and of little concern, some reports have drawn attention to rare life-threatening reactions. One of the most dangerous of theses is acute pulmonary oedema.

Steinberg first described acute pulmonary oedema due to HCTZ in $1968^{[1]}$, with around 50 other cases so far reported in the literature. We here report a case of acute pulmonary oedema following ingestion of ramipril/HCTZ. 


\section{CASE REPORT}

A 73-year-old woman presented to the Emergency Department (ED) following the sudden onset of acute respiratory failure. Her past medical history included long-lasting hypertension, permanent atrial fibrillation and mitral stenosis, which was reportedly the consequence of rheumatic heart disease during childhood. Her latest echocardiography results showed an ejection fraction of $60 \%$ with normal diastolic function, no alteration of left ventricular wall motion and mild tricuspid regurgitation. She had recently undergone her annual cardiological follow-up examination, which had proven unremarkable apart from suboptimal blood pressure control. The cardiologist had therefore suggested switching from amlodipine to a combination tablet containing ramipril and HCTZ. The following day, 20 min after taking the new drug, fever, vomiting and diarrhoea occurred, followed by severe dyspnoea. On evaluation in the ED, the patient was found to have acute respiratory failure. Physical examination was notable for mild hypotension, fever and a respiratory rate of 30 breaths per minute with low levels of peripheral oxygen saturation; ECG showed sinus rhythm. Arterial blood gas (ABG) showed mixed respiratory and metabolic acidaemia. The chest radiograph was consistent with fluid overload. A complete blood count revealed leucopenia and a high level of haemoglobin, which was attributed to haemoconcentration (Table 1). A diagnosis of acute pulmonary oedema was made and the patient was admitted to the High Dependency Unit (HDU) where non-invasive ventilation (NIV) and intravenous nitrate infusion were started. After 2 hours of NIV, the ABG improved and respiratory support was switched to low-flow oxygen administration by nasal cannula. During the remainder of her stay in the HDU, the patient reported no other symptoms. Considering the timing of onset, the normal systolic and diastolic function and the absence of hypertension, the hypothesis of pulmonary oedema due to an idiosyncratic reaction to HCTZ was proposed. Consequently, the antihypertensive therapy was modified and the patient was discharged home with a good performance status.

\begin{tabular}{|l|c|c|}
\hline \multicolumn{1}{|c|}{ Blood test } & $09 / 06 / 2017$ & $11 / 06 / 2017$ \\
\hline WBC $\left(4-10 \times 10^{3} \mu l\right)$ & 2070 & 8360 \\
\hline Hb $(12.5-16 \mathrm{~g} / \mathrm{dl})$ & 17.1 & 14.4 \\
\hline HCT $(38-48 \%)$ & 51.6 & 42.4 \\
\hline PIts $\left(150-450 \times 10^{3} \mu l\right)$ & 152 & 129 \\
\hline PCR $(<0.5 \mathrm{mg} / \mathrm{dl})$ & $<0.5$ & $\mathrm{ND}$ \\
\hline PCT $(\mathrm{ng} / \mathrm{ml})$ & $\mathrm{ND}$ & 6.24 \\
\hline Creatinine $(0.5-0.9 \mathrm{mg} / \mathrm{dl})$ & 1 & 0.9 \\
\hline TnT $(<0.05 \mathrm{ng} / \mathrm{ml})$ & 0.03 & $\mathrm{ND}$ \\
\hline
\end{tabular}

Table 1. Results of patient blood tests

\section{DISCUSSION}

Acute pulmonary oedema is traditionally classified as 'cardiogenic', related to an increase in pulmonary wedge pressure, or 'non-cardiogenic', caused by increased vascular permeability ${ }^{[2]}$. Non-cardiogenic pulmonary oedema is a syndrome characterized by severe hypoxaemia and bilateral alveolar infiltrates on the chest radiograph, without evidence of heart failure ${ }^{[2]}$. Causes of non-cardiogenic pulmonary oedema include infections, trauma, disseminated intravascular coagulation and drugs ${ }^{[2]}$. Acute pulmonary oedema induced by $\mathrm{HCTZ}$ seems to belong to the non-cardiogenic type.

The reaction can develop either following the first administration of the drug or during the course of treatment. In general, patients develop symptoms $10-150$ min after taking HCTZ, as in our patient ${ }^{[3]}$. While the vast majority of patients (90\%) report predominantly respiratory symptoms (eg, dyspnoea, wheezing, cough), 54\% also experience gastrointestinal symptoms. In this case, pulmonary oedema, fever and gastrointestinal symptoms were all present ${ }^{[4,5]}$. In line with the general assumption of a female predominance in adverse drug reactions, more than $90 \%$ of all case reports involve women ${ }^{[4,5,6]}$. Interestingly, procalcitonin levels were high without signs of infections. This well-described trend of procalcitonin has no clear explanation and rises doubts about its specificity in acute critical situations ${ }^{[6]}$. Although some patients received corticosteroids, the use of these agents is not supported by clinical evidence. Most patients respond to supportive treatment and discontinuation of the offending drug, with symptoms resolving, on average, in 3 days.

The pathogenesis of this rare life-threatening side effect is still uncertain. Diagnostic tests, including skin testing, patch testing and lymphocyte transformation tests (LTT), have not been shown to be predictive. Reports of reaction occurring after the first administration of the drug do not support an acquired immunological basis ${ }^{[7]}$; although an oral challenge would be useful to determine causality, re-challenge with a thiazide diuretic is not recommended because of the rapid and severe onset of symptoms after re-exposure. Neither cellular nor humoral 
immunological mechanisms could be demonstrated in this patient with standard techniques, suggesting an idiosyncratic reaction ${ }^{[4-7]}$. Some authors support the hypothesis of a type 1 hypersensitivity reaction based on immunoglobulin E-mediated basophil activation. However, allergic symptoms such as urticarial rash, angioedema and bronchospasm, have never been described either in reported series or in our case ${ }^{[8]}$.

Another proposed mechanism is a type 3 hypersensitivity reaction, characterized by accumulation of antigen-antibody immune complexes. According to this theory, anti-HCTZ IgG immune complexes develop, giving rise to a systemic inflammatory response. Acute lung oedema would occur at the time of immune complex deposition in the alveolar membranes ${ }^{[8]}$. Nevertheless, such a mechanism appears less probable since the reaction seems limited to HCTZ with no cross-reactivity with other thiazide drugs ${ }^{[8]}$.

The most widely accepted hypothesis is an idiosyncratic response, similarly to other drug-induced reactions. The initially low white blood cell count, seen both in our patient and in other previously reported cases, is consistent with intrapulmonary sequestration of granulocytes leading to pulmonary oedema. In support of this hypothesis, previous studies have demonstrated a large percentage of neutrophils in the lung. Therefore, the HCTZ-induced acute lung injury is more probably due to a neutrophil-mediated reaction than to a type 1 or 3 hypersensitivity reaction ${ }^{[8]}$.

\section{CONCLUSION}

Non-cardiogenic pulmonary oedema is a rare reaction to HCTZ ingestion. As a result, the possibility of HCTZ-induced pulmonary oedema is not routinely considered in the differential diagnosis of pulmonary oedema. If a middle-aged or older patient presents with acute dyspnoea and hypoxia, the physician must rule out the possibility of acute coronary syndrome or pulmonary embolism before considering other less common conditions. However, since HCTZ is one of the most popular drugs for the treatment of hypertension and millions of patients take it on a daily basis, it is important to keep in mind not only the common adverse reactions but also the most dangerous ones, even if rare. This complication has mostly been reported in women with an average age of 56 years; the average time to develop this complication is 45 min after taking a thiazide pill. In conclusion, physicians who prescribe HCTZ should be familiar with this life-threatening adverse reaction and should share this information with their patients, advising them to seek immediate medical attention if they experience complications.

\section{REFERENCES}

1. Steinberg AD. Pulmonary edema following ingestion of hydrochlorothiazide. JAMA 1968;204:825-827.

2. Hackam DG, Khan NA, Hemmelgarn BR, et al. The 2010 Canadian Hypertension Education Program recommendation for the management of hypertension: part 2-therapy. Can J Cardiol 2010;26:249-258.

3. Fine SR, Lodha A, Zoneraich S, et al. Hydrochlorothiazide induced acute pulmonary edema. Ann Pharmacother 1995;29:701-703.

4. Mas A, Jordana R, Vallès J, et al. Recurrent hydrochlorothiazide-induced pulmonary edema. Intensive Care Med 1998;24:363-365.

5. Andresen M, González A, Espino A, et al. Thiazide induced acute pulmonary edema: report of one case. Rev Med Chile 2007;135:496-500.

6. Goetschalckx, K, Ceuppens, J, Van Mieghem W. Hydrochlorothiazide-associated noncardiogenic pulmonary oedema and shock: a case report and review of the literature. Acta Cardiol 2007;62:215-220.

7. Darwish OS, Criley J. Hydrochlorothiazide-induced noncardiogenic pulmonary edema: BAL fluid analysis. Chest 2011;139:193-194.

8. Bernal C, Patarca R. Hydrocholorotiazide-induced pulmonary edema and associated immunologic changes. Ann Pharmacother 1999;33:172-174. 